Classification

Physics Abstracts

$6.50-7.80-78.70$

\title{
Quantitative Microanalysis Using Electron Energy-Loss Spectrometry: II. Compounds with Heavier Elements
}

\author{
Ferdinand Hofer and Gerald Kothleitner \\ Forschungsinstitut für Elektronenmikroskopie, Technische Universität Graz, Graz, 8010, Austria
}

(Received November 5, 1996; accepted January 6, 1997)

\begin{abstract}
Electron energy-loss spectrometry (EELS) in the TEM can be used for the quantitative analysis of compounds including both light and heavy elements at a submicrometre scale. However, EELS-quantification can become complicated due to low edge-to-background ratios, problems with multiple scattering in case of thicker samples, inaccuracies due to background extrapolation or edgeoverlapping. All these problems can be overcome by careful use of well known procedures. A further problem of quantitative EELS is the need for precise partial ionization cross-sections that are sometimes not well known. In this work, we have quantified EELS-spectra of compounds with known composition: $\mathrm{LaCoO}_{3}, \mathrm{YBa}_{2} \mathrm{Cu}_{3} \mathrm{O}_{7}$ and $\mathrm{LaB}_{6}$. To demonstrate the achievable accuracy with different models, the quantifications have been calculated with theoretical cross-sections (hydrogenic model and Hartree-Slater model) and experimentally determined $k$-factors. In every single case more accurate quantification results could be obtained when experimentally derived cross-sections (or $k$-factors) were used, giving concentration values that lie within 5 rel\% of the nominal composition.
\end{abstract}

\section{Introduction}

Within the last few years, electron energy-loss spectrometry (EELS) in an analytical electron microscope (AEM) has found numerous applications in the materials and biological sciences [1]. EELS enables the compositional analysis of chemical elements on a submicrometre scale which can be identified because of their characteristic ionization edges. It is well known that EELS is a powerful tool for the detection and quantitative analysis of light elements. But EELS can also be used for the detection of heavy elements quite efficiently, e.g. first and second row transition elements and the rare earth elements $[2,3]$.

Although EDX-spectrometry of thin films yields better analytical sensitivities for high $Z$ elements, there are often severe problems in case of compounds consisting both of light and heavy elements, e.g. when overlapping X-ray lines and self absorption of soft X-rays render quantitative analysis difficult. Other analytical techniques such as Auger spectroscopy and secondary ion mass spectrometry are very sensitive to the presence of light and heavy elements, but suffer from 
the drawback that they lack the spatial resolution of EELS, being in favourable cases less than some nm $[4,5]$.

Although EELS quantification procedures have been established some years ago [1], EELS is mostly used as a qualitative tool. This is due to several problems that remain in the process of extracting absolute or relative concentrations of a given atomic species from the raw spectral data. The limitations originate from several sources:

1) Specimen thickness. Results from EELS analysis are thickness dependent. Therefore, spectra should be taken only from very thin specimen regions, otherwise spectra must be deconvoluted in order to remove multiple scattering contributions (see [1]).

2) Intensity determination. The continuously decreasing background must be subtracted from the signal of interest for each element. Although the high-energy tail generally approximates to a power-law energy dependence $\left(A E^{-r}\right)$, problems can arise in case of low energy edges. So alternative background subtractions have been proposed particularly for the low-loss edges where $A$ and $r$ are varying more than at higher energy-losses [6, 7]. Other problems arise from the overlapping of closely spaced edges: the "separation" of these edges is a particular problem and limits the accuracy of quantification results.

3) Ionization cross-sections. In order to convert the edge intensities into elemental concentrations it is necessary to know the ionization cross-section for the relevant ionization edges either absolutely or relative to those of other elements present. The ionization cross-sections can be either calculated on the basis of the Hartree-Slater [8,9] or the hydrogenic model $[10,11]$, or by measurement from standard samples like binary oxides [12,13]. Although the knowledge on ionization cross-sections has been enormously increased during the last ten years $[14,15]$, there are still some uncertainties especially in the low energy-loss region and with heavy elements. Since in the meantime there is some confusion which cross-section data should be given preference, we will discuss this aspect thoroughly.

In a first paper we have shown that $\mathrm{Li}$ - and Be-oxides and silicates can be accurately quantified with EELS [16]. In this paper, we present further practical examples for EELS-quantification which is in continuous development in our laboratory. We will describe the quantitative analysis of compounds containing both light and heavy elements. Despite the great importance of such compounds for materials science, EELS has been only scarcely applied for quantitative analysis.

\section{Experimental Procedures}

All samples were prepared by crushing selected crystals in alcohol and pipetting the suspension onto holey carbon grids. The spectra were measured using a Philips CM20 transmission electron microscope equipped with $\mathrm{LLB}_{6}$ source; the TEM was fitted with a Gatan Imaging Filter which incorporates a slow-scan-CCD camera for detection $(1 \mathrm{M} \times 1 \mathrm{M})$ [17]. The GIF has been operated in spectrum mode. To prevent saturation of the slow-scan CCD camera, the spectrum incident on the detector is spread in the non-dispersion direction over 100 CCD pixels and spectrum profiles were obtained by lateral integration over the pixels. This procedure gives better sensitivity and an essentially better dynamic range than a one dimensional photodiode array such as used in conventional PEELS-systems [18]. For spectroscopy the TEM was operated at a primary energy of $200 \mathrm{kV}$ in image mode; screen magnification was usually within the range of $3000-10000 \times$. Beam convergence was lower than $1.5 \mathrm{mrad}$ and the collection semi-angle $\beta$, defined by the microscope objective aperture, was set to $7.6 \mathrm{mrad}$. The analyzed areas were defined by a $3 \mathrm{~mm}$ spectrometer entrance aperture. Spectra acquisition times were rather short for the GIF-PEELS system, typically $5 \mathrm{~s}$ for the low loss part with the oxygen K-edge and usually $30 \mathrm{~s}$ for the high energy loss part, e.g. the $\mathrm{Y} \mathrm{L}_{23}$-edge. Since the spectra were sampled with a dispersion of $0.5 \mathrm{eV}$ per channel 
and with 1024 channels per readout, we recorded two spectra in some cases which were scaled together afterwards: beginning with the low loss region and the low energy edges, we subsequently recorded the higher energy edges (e.g. $\mathrm{Y} \mathrm{L}_{23}$ edge).

\section{EELS Data Processing}

Each spectrum was corrected for dark current and intrinsic channel to channel gain variations between the 1024 detector elements of the CCD. We used Gatan's EL/P 3.0 program package for further processing of the spectra such as deconvolution, computation of $t / \lambda$-values, fitting of the background, determination of edge intensities and calculation of HS values.

3.1 SpeCIMEN ThICKNESS. - Detection sensitivity and validity of EELS quantification in thicker samples is limited severely by multiple scattering. Therefore spectra were generally taken at the thinnest edges of the crushed crystals. Specimen thickness $t$ was determined from the energy-loss spectra with the plasmon intensity method as follows [1]:

$$
t=\lambda \ln \left(I_{\mathrm{t}} / I_{0}\right)
$$

where $I_{0}$ and $I_{\mathrm{t}}$ are the integrated intensities in the zero loss peak and the total intensity in the spectrum, respectively. The mean free path of inelastic scattering $\lambda$ may be estimated from the energy dependence of the low-loss spectrum, utilizing a Kramers-Kronig sum rule [1].

3.2 Deconvolution of The Spectra. - Although the analyzed specimen regions were thinner than one quarter of the mean free path for inelastic scattering $(\lambda)$, some spectra had to be deconvoluted for removing plural-scattering features before background subtraction and quantification. This was carried out with the Fourier-log method [1] which improves the edge-to-background ratio and the background in front of the edges thus making background subtractions more reliable.

However, it is worth noting that for an artefact-free deconvolution there should be good linearity of the electron counting system in addition, spectra should be recorded with a large collection angle from samples of uniform thickness [19]. On the other hand small collection angles guarantee a good peak-to-background ratio in the low loss-region. As a compromise we have chosen a medium collection angle of $7.6 \mathrm{mrad}$. Experimental results [20] indicate that the Fourier-logmethod is accurate to better than $3 \%$ in the low-loss region, for apertures commonly used in a TEM. Since deconvolution artifacts increase in case of thicker samples [1], spectra were always collected from specimen regions not thicker than about $0.5 \lambda$. Additionally the spectra have been taken from specimen regions with small thickness variations.

3.3 BACKGRound SubTraction. - Numerous background subtraction techniques have been proposed to extract intensities "correctly". Their use depends on the shape of the EELS-spectrum and/or the energy of the edge under consideration. However, reliable background subtractions have been found with the power-law background model for most ionization edges. These findings agree with the work of Bonnet et al. [21], who compared different background subtraction methods and found that the classical $A E^{-r}$ fit gives the best results in every case. Consequently, in this work backgrounds below the edges were determined by fitting a power-law curve $\left(I=A E^{-r}\right.$, where $A$ and $r$ are two fitting parameters) to a selected background region just ahead of the edge: in case of low-loss edges only 20-30 eV windows were used, whereas for the high-loss edges windows up to a width of $200 \mathrm{eV}$ are preferable. 
3.4 MLS-Fit of Overlapping Edges. - As already discussed, a great limitation to EELS analysis is that imposed by overlapping peaks. Edge intensities then can only be determined by separation with a set of reference spectra fitted to the unknown by a multiple-least-squares (MLS) algorithm. This method is similar to that used in the analysis of energy-dispersive X-ray spectra. Such a procedure for the application in EEL spectrometry has been first proposed by Shuman and Somlyo [22]: in this case the background underlying the group of edges was removed by computing the first difference spectrum so that the slowly varying background is strongly suppressed. Provided the reference spectra are treated similarly they can be fitted directly to the unknown spectrum. Leapman and Swyt have demonstrated that this method can be applied to undifferentiated spectra as well, if the intensity in front of the group of edges is extrapolated and subtracted in the conventional way [23]. A similar approach has been used by Manoubi et al. [24].

The spectrum with the overlapping edges is quantified with a multiple least squares fit to reference spectra applying a singular value decomposition (SVD) technique $[25,26,16]$. If the $k$ th reference spectrum is labelled $X_{k}\left(E_{i}\right)$ where $k=1$ to $M$, then the fitting coefficients $a_{k}$ are determined by minimizing the value of $\chi^{2}$,

$$
\chi^{2}=\frac{\sum_{i=1}^{N}\left[I\left(E_{i}\right)-\sum_{k=1}^{M} a_{k} * X_{k}\left(E_{i}\right)\right]^{2}}{\left[\sigma\left(E_{i}\right)\right]^{2}}=\text { minimum. }
$$

$I\left(E_{i}\right)$ is the intensity recorded at the $i$ th channel $(i=1$ to 1024$)$ at energy-loss $E_{i}$ and $\sigma\left(E_{i}\right)$ is the standard error in the $i$ th channel of the unknown spectrum.

The reference spectra - thin film standards have been used - have been measured under identical conditions as the unknown spectra. They have to be treated in a similar way, i.e. Fourier-logdeconvolution and conventional background subtraction. Deconvolution of the references and the unknown is necessary in our approach, because plural inelastic scattering changes the edge shapes and the MLS-fit can fail. Another possibility to take into account plural scattering has been proposed by Leapman and Swyt [23]. In this case the single scattering reference edges are additionally convolved with the low loss spectrum of the unknown. However, in case of the lowloss edges the first approach seems to be more useful because the Fourier-log-deconvolution not only improves the behaviour of the background below the edges but also enhances the visibility of edges. This has a favorable effect especially on the low-loss region, where edge to background ratios are generally very small.

The MLS method was derived on the assumption that edge profiles are the same in the unknown and in the reference. Since variations in chemical bonding can significantly modify edge fine structures [27], we used oxide standards with the same coordinations of the metal atom. Then ELNES should be largely similar.

Since the goodness of the fit is also a function of energy-loss calibration of the unknown spectrum with respect to the references, quite large errors can occur even if there is a miscalibration of $1 \mathrm{eV}$. To correct these miscalibrations and to account for different chemical shifts of the references and the unknown spectra, the fitting program allows for the shift of the references relative to the edge thresholds of the measured spectrum. This feature essentially improves the fit of the unknown spectrum.

3.5 Quantification. - The atomic concentration ratio $N_{\mathrm{A}} / N_{\mathrm{B}}$ of any elements $\mathrm{A}$ and B may be determined from an EEL spectrum using the following equation [1]

$$
\frac{N_{\mathrm{A}}}{N_{\mathrm{B}}}=\frac{I_{\mathrm{A}}(\beta, \Delta)}{I_{\mathrm{B}}(\beta, \Delta)} \frac{\sigma_{\mathrm{B}}(\beta, \Delta)}{\sigma_{\mathrm{A}}(\beta, \Delta)}
$$


where $N_{i}$ is the number of atoms per unit area, $I_{i}(\beta, \Delta)$ is the core loss intensity integrated up to an energy region of width $\Delta$ starting at the edge onset, and $\sigma_{i}(\beta, \Delta)$ is the partial ionization cross-section within an acceptance angle $\beta$ and an energy region $\Delta$.

The partial cross-sections can be calculated using the hydrogenic model [1] and the Hartree-Slater model [9] (see Tab. I). The hydrogenic model algorithm has been incorporated into short computer programs called SIGMAK [10] for the K-edges (elements $\mathrm{Li}$ to Si) and SIGMAL [11] for the $\mathrm{L}_{23}$ edges (elements $\mathrm{Al}$ to $\mathrm{Zn}$ ). Two versions of the SIGMAL-program are used today; the older one does not take into account the white lines at the thresholds of the elements $\mathrm{K}$ to $\mathrm{Ni}$ [11], the second version includes these white lines [1], because the model has been readjusted to better consider photoabsorption data and to take into account energy-loss measurements. Recently a hydrogenic model for $\mathrm{M}_{45^{-}}, \mathrm{N}_{45^{-}}$and $\mathrm{M}_{23}$-shells has also been developed, applying a correction based on experimental photoabsorption data [28]. The Hartree-Slater model can be used for all kind of ionization edges (K-, $\mathrm{L}_{23^{-}}, \mathrm{M}_{45^{-}}, \mathrm{N}_{45^{-}}, \mathrm{M}_{23}$-edges) thus enabling the quantification of nearly all elements. The program is also available in two versions; the older version which is currently included in the EL/P program does not take into account white lines [9]. Recently, oscillator strengths for the white-line components of L- and M-edges have been calculated, which can be used to generate partial cross-sections including both bound and continuum states [29].

Additionally we used experimental $k$-factors which can be comfortably used instead of crosssections, since they are cross-section ratios which are measured relative to the $\mathrm{K}$ edge of oxygen, e.g. for the element A:

$$
k_{\mathrm{A}, \mathrm{O}}=\frac{\sigma_{0}(\Delta, \beta)}{\sigma_{\mathrm{A}}(\Delta, \beta)} .
$$

The $k$-values for the $\mathrm{L}_{23^{-}}, \mathrm{M}_{45^{-}}$and $\mathrm{N}_{45}$-edges were taken from previously published measurements at $120 \mathrm{kV}$ [13] and have been converted into $200 \mathrm{kV} k$-factors with programs proposed by Egerton [30]. This can be achieved via integrated dipole oscillator strengths, having the advantage to be independent of the experimental conditions $\left(E_{0}, \beta\right)$. The $k$-factors for the $\mathrm{M}_{23}$-edge and the $\mathrm{Y} \mathrm{L}_{23}$-edge were taken from recently published measurements performed directly at $200 \mathrm{kV}$ $[31,32]$.

\section{Results}

4.1 $\mathrm{LaCoO}_{3}$. - This oxide belongs to the perowskite group and shows like many other perowskites interesting properties [33]. In order to adjust their macroscopic properties these ceramic materials are often doped with a wide range of elements. Consequently, quantification of these materials on a micro- and also nanoscale is important. We have chosen $\mathrm{LaCoO}_{3}$ as a demanding example, where the quantification is difficult because of overlapping ionization edges. Oxide compounds containing first row transition elements and rare earth elements exhibit two kinds of ionization edges: the Co $\mathrm{M}_{23}$ - and the $\mathrm{La} \mathrm{N}_{45}$-edges in the low-loss region and the $\mathrm{Co} \mathrm{L}_{23}$ - and the $\mathrm{La} \mathrm{M}_{45}$-edges in the high-loss region.

Figure 1a shows the relevant parts of the energy-loss spectrum of $\mathrm{LaCoO}_{3}$ with the $\mathrm{O} \mathrm{K}$-edge together with the overlapping $\mathrm{Co}_{23}$ - and $\mathrm{La} \mathrm{M} 45$-edges $(t / \lambda=0.45)$. This spectrum was first Fourier-log deconvoluted to provide an adequate base for MLS-fitting and to improve the visibility of the edges (Fig. 1b). As already mentioned in Section 3.4, it would be possible to perform the fit on first difference spectra. In this case background subtraction is unnecessary because slowly varying features are cancelled out.

The background region in front of the edge was modelled using the power-law $\left(A E^{-r}\right)$ with a pre-edge fitting area of $80 \mathrm{eV}$ immediately beginning at the threshold of the $\mathrm{Co}_{23}$-edge. The MLS-fit was performed with the Fourier-deconvoluted reference edges from $\mathrm{Co}_{3} \mathrm{O}_{4}$ and $\mathrm{La}_{2} \mathrm{O}_{3}$ 
Table I. - Partial cross-sections and $k$-factors for the ionization edges used for the quantifications. Experimental conditions: $E_{0}=200 \mathrm{kV}, \beta=7.6 \mathrm{mrad}, \alpha=1.5 \mathrm{mrad}$.

\begin{tabular}{|c|c|c|c|c|c|}
\hline \multirow[b]{2}{*}{ Edge } & \multicolumn{3}{|c|}{$\sigma(\beta, \Delta)$} & \multicolumn{2}{|c|}{$k$-factors } \\
\hline & $\begin{array}{c}\text { HS-model } \\
{[8,9]}\end{array}$ & $\begin{array}{c}\text { Hydr.-model } \\
\text { with w.1. } \\
{[1,28]}\end{array}$ & $\begin{array}{c}\text { Hydr.-model } \\
\text { without w.l. } \\
{[11]}\end{array}$ & $\begin{array}{c}\text { recalc. from } \\
\text { exp. } 120 \mathrm{kV} \\
\text { data }[13]\end{array}$ & $\begin{array}{c}\text { exp. } 200 \mathrm{kV} \text { - } \\
\text { data } \\
{[31,32]}\end{array}$ \\
\hline $\begin{array}{c}\mathrm{OK} \\
(50 \mathrm{eV})\end{array}$ & $6.57 \mathrm{E}-22$ & $7.43 \mathrm{E}-22$ & & & \\
\hline $\begin{array}{c}\mathrm{Co} \mathrm{L}_{23} \\
(50 \mathrm{eV})\end{array}$ & 7.83 E-22 & 9.98 E-22 & 7.24 E-22 & 0.845 & -- \\
\hline $\begin{array}{l}\mathrm{La} \mathrm{M}_{45} \\
(50 \mathrm{eV})\end{array}$ & $1.23 \mathrm{E}-21$ & $1.48 \mathrm{E}-21$ & -- & 0.274 & -- \\
\hline $\begin{array}{l}\mathrm{Co} \mathrm{M}_{23} \\
(30 \mathrm{eV})\end{array}$ & $1.36 \mathrm{E}-20$ & $1.13 \mathrm{E}-20$ & --- & --- & $5.70 \mathrm{E}-3$ \\
\hline $\begin{array}{c}\mathrm{La} \mathrm{N}_{45} \\
(30 \mathrm{eV})\end{array}$ & $2.74 \mathrm{E}-20$ & $1.15 \mathrm{E}-20$ & --- & $2.93 \mathrm{E}-3$ & -- \\
\hline $\begin{array}{l}\mathrm{Ba} \mathrm{M}_{45} \\
(50 \mathrm{eV})\end{array}$ & $2.15 \mathrm{E}-21$ & $1.83 \mathrm{E}-21$ & & 0.288 & \\
\hline $\begin{array}{c}\mathrm{Cu} \mathrm{L}_{23} \\
(50 \mathrm{eV})\end{array}$ & $5.24 \mathrm{E}-22$ & $4.33 \mathrm{E}-22$ & $4.48 \mathrm{E}-22$ & 2.56 & \\
\hline $\begin{array}{c}\mathrm{Y} \mathrm{L}_{23} \\
(100 \mathrm{eV})\end{array}$ & $6.42 \mathrm{E}-23$ & 7.01 E-23 & & & 21.4 \\
\hline $\begin{array}{c}\mathrm{La} \mathrm{N}_{45} \\
(50 \mathrm{eV})\end{array}$ & $3.19 \mathrm{E}-20$ & $1.50 \mathrm{E}-20$ & --- & $3.97 \mathrm{E}-3$ & -- \\
\hline $\begin{array}{c}\text { B K } \\
(50 \mathrm{eV})\end{array}$ & $6.49 \mathrm{E}-21$ & $8.01 \mathrm{E}-21$ & --- & 0.102 & -- \\
\hline
\end{tabular}

and it is obvious that both edges are fitted quite well. To quantify the spectrum from Figure $1 \mathrm{~b}$ the intensities of the reference edges were integrated within $50 \mathrm{eV}$ above the edge onset. The quantification result is shown in Table II clearly demonstrating that experimental $k$-factors [13] give better agreement with the nominal concentration ratios than the hydrogenic model (SIGMAL2) and Hartree-Slater models. Calculating the O/Co ratio with SIGMAL2 (hydrogenic model with white line correction) the nominal value (3.0) is overestimated (3.55), whereas ratio determinations with SIGMAL1 (without white line correction) leads to lower values, deviating in the other direction (2.5, not displayed).

In addition the low-loss edges can be also used to determine the chemical composition of the specimen. However, quantification is troublesome since these edges are situated close to the intense plasmon peak and hence exhibit a very low signal-to-background ratio. Figure 2 shows the low-loss spectrum of the $\mathrm{LaCoO}_{3}$-specimen including the $\mathrm{Co}_{23}$ - and the $\mathrm{La} \mathrm{N}_{45}$ edges. Although the spectrum shown in Figure 2 has been recorded from a thin specimen region $(t / \lambda=0.26)$, deconvolution with the Fourier-log method was called for since the signal to 


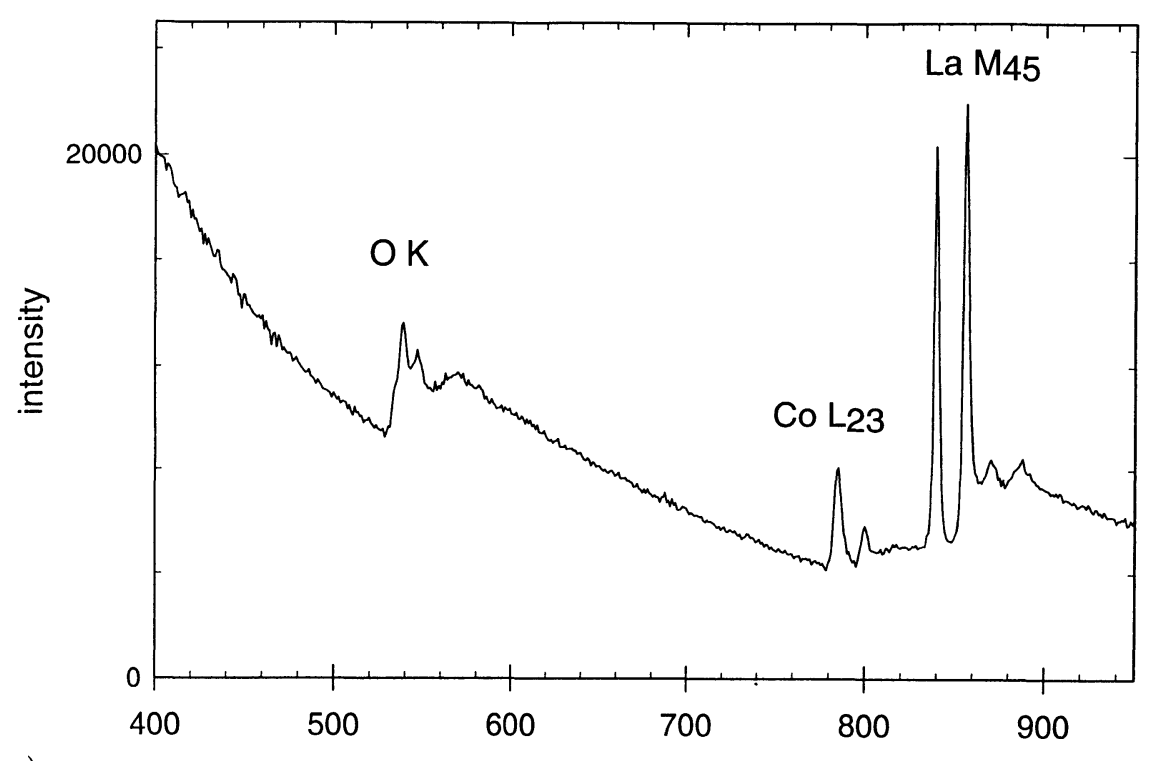

a)

energy loss [eV]

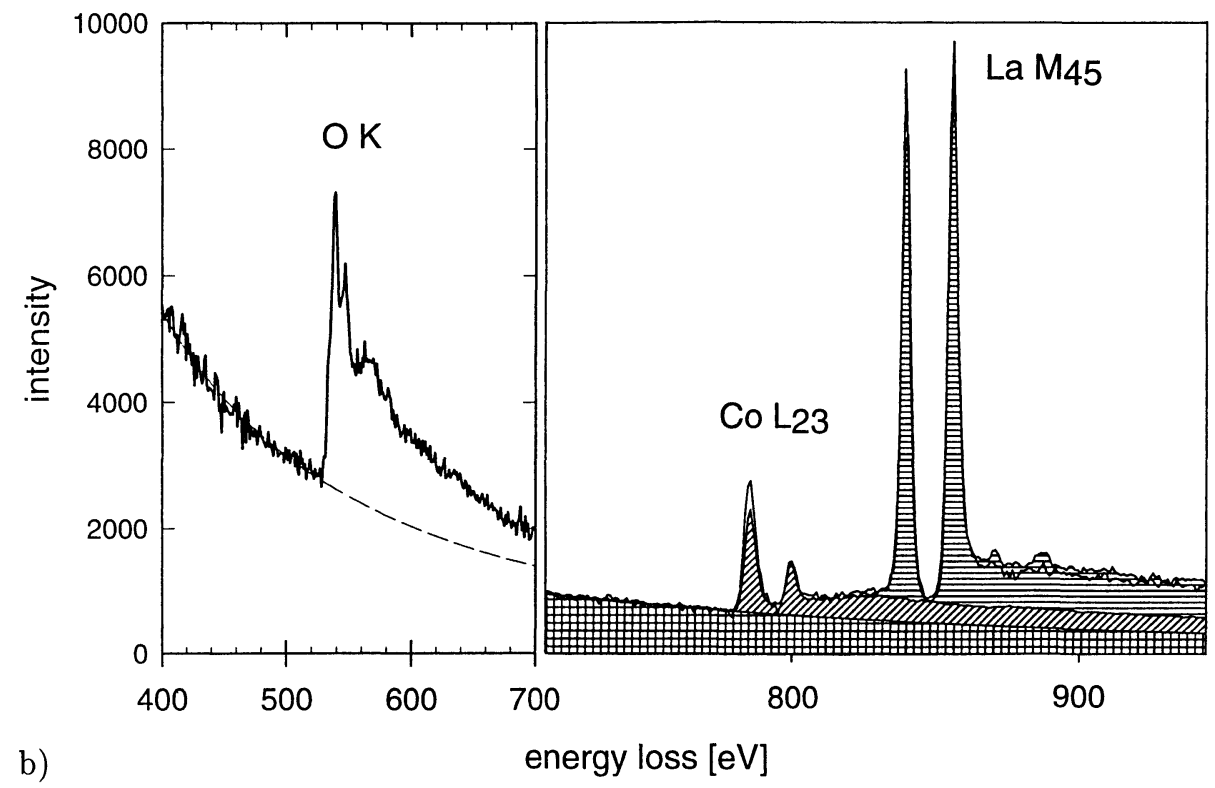

Fig. 1. - EEL-spectrum of a $\mathrm{CoLaO}_{3}$ crystal: a) original spectrum $(t / \lambda=0.45)$, b) deconvoluted with the Fourier-log-method and MLS-fit with reference edges.

background ratio for these low-energy edges can be essentially improved [16]. The backgrounds before the edges were fitted over a region of $20 \mathrm{eV}$ again applying the power-law model $\left(A E^{-r}\right)$. Quantification results are presented in Table II. For the Hartree-Slater model and the experimental $k$-factors the $\mathrm{Co} / \mathrm{La}$ ratio is in good agreement with the nominal value of 1.0 . However, hydrogenic model cross-sections [28] underestimate the Co/La-ratio by a factor of 2 . 
Table II. - Results of EELS quantifications of some inorganic compounds; atomic ratios have been determined using either experimentally determined $k$-factors and calculated cross-sections. Experimental conditions: $E_{0}=200 \mathrm{kV}, \beta=7.6 \mathrm{mrad}, \alpha=1.5 \mathrm{mrad}$; average values for 5 spectra with standard deviation.

\begin{tabular}{|c|c|c|c|c|c|}
\hline Compound & at.-ratio & nominal & exp. $k$-factors & HS-model & hydr. model \\
\hline $\mathrm{LaCoO}_{3}$ & $\mathrm{O} / \mathrm{La}$ & 3.0 & $2.93 \pm 0.35$ & $1.50 \pm 0.18$ & $1.60 \pm 0.19$ \\
\hline $\mathrm{La} \mathrm{M}_{45}$ & $\mathrm{O} / \mathrm{Co}$ & 3.0 & $3.14 \pm 0.22$ & $3.16 \pm 0.22$ & $3.55 \pm 0.25$ \\
\hline $\mathrm{CoL}_{23}$ & $\mathrm{Co} / \mathrm{La}$ & 1.0 & $0.93 \pm 0.15$ & $0.47 \pm 0.07$ & $0.45 \pm 0.07$ \\
\hline $\begin{array}{c}\mathbf{L a C o O}_{3} \\
\mathrm{La} \mathrm{N}_{45}, \mathrm{CoM}_{23}\end{array}$ & $\mathrm{Co} / \mathrm{La}$ & 1.0 & $1.04 \pm 0.09$ & $1.08 \pm 0.10$ & $0.55 \pm 0.05$ \\
\hline \multirow{3}{*}{$\begin{array}{c}\mathbf{Y B a}_{2} \mathbf{C u}_{3} \mathbf{O}_{7} \\
\mathrm{YL}_{23}, \mathrm{Ba} \mathrm{M}_{45} \\
\mathrm{Cu} \mathrm{L}_{23}\end{array}$} & $\mathrm{O} / \mathrm{Y}$ & 7.0 & $7.41 \pm 0.6$ & $8.48 \pm 0.7$ & $10.6 \pm 0.9$ \\
\hline & $\mathrm{O} / \mathrm{Ba}$ & 3.5 & $3.29 \pm 0.15$ & $3.10 \pm 0.14$ & $2.33 \pm 0.1$ \\
\hline & $\mathrm{O} / \mathrm{Cu}$ & 2.33 & $2.26 \pm 0.13$ & $4.63 \pm 0.27$ & $3.38 \pm 0.2$ \\
\hline $\begin{array}{c}\mathbf{L a B}_{\mathbf{6}} \\
\mathrm{La} \mathrm{N}_{45}, \mathrm{~B} \mathrm{~K}\end{array}$ & $\mathrm{~B} / \mathrm{La}$ & 6.0 & $5.46 \pm 0.2$ & $8.03 \pm 0.04$ & $0.39 \pm 0.03$ \\
\hline
\end{tabular}

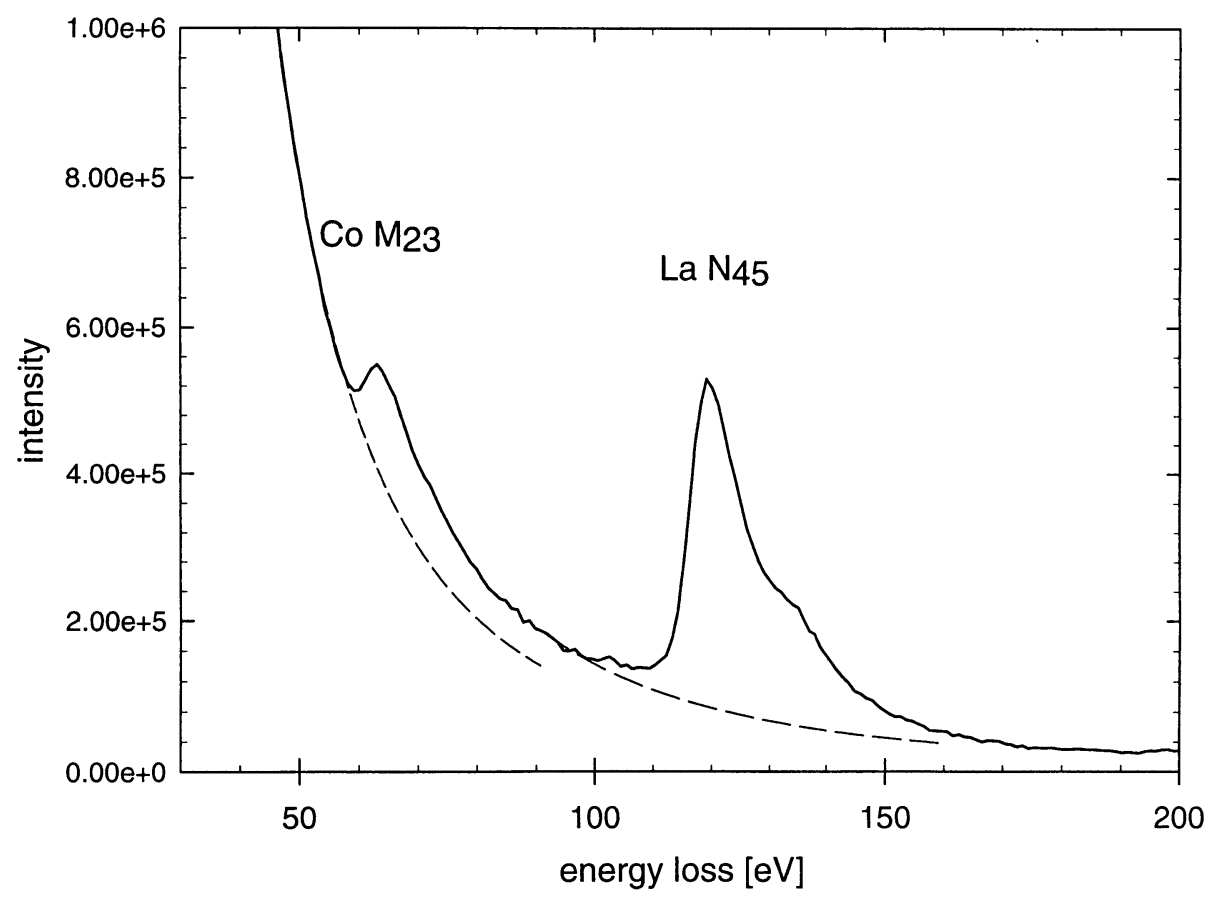

Fig. 2. - EEL spectrum of a $\mathrm{CoLaO}_{3}$ crystal; low-loss region with the $\mathrm{Co}_{23}$ - and $\mathrm{La} \mathrm{N}_{45}$-edges; spectrum has been deconvoluted with the Fourier-log method; $t / \lambda=0.26$; background fits are indicated. 


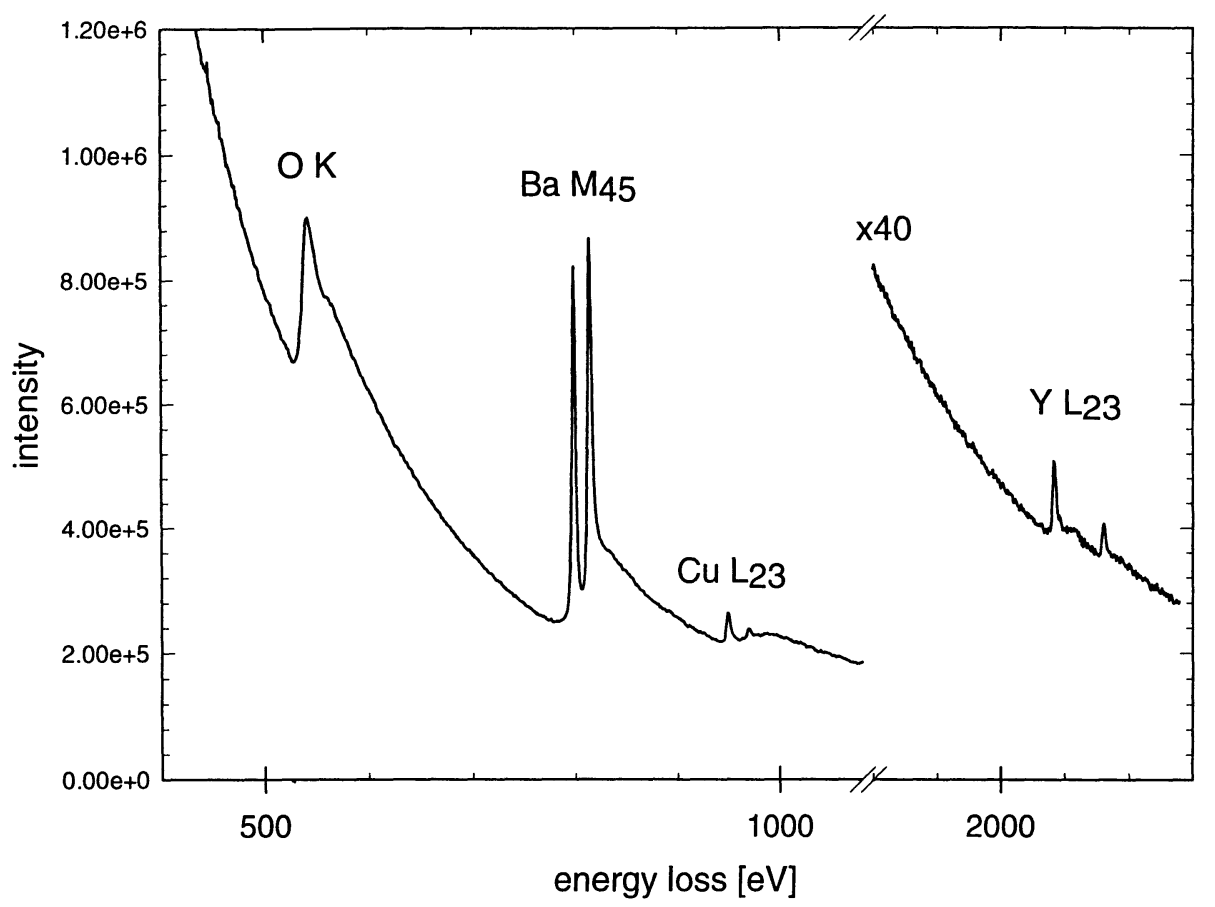

Fig. 3. - EEL-spectrum of a $\mathrm{YBa}_{2} \mathrm{Cu}_{3} \mathrm{O}_{7}$-sample; $(t / \lambda=0.20)$.

4.2 Y-Ba-Cu-OxIDE. - This already well known compound [34] has been investigated by EELS for several times, but accurate EELS-quantification has been only scarcely performed. This is probably due to difficulties in extracting the Y-signal, because the commonly used $\mathrm{Y} \mathrm{M}_{45}$-edge has a low signal-to-background ratio. However, this problem can be overcome by using the $\mathrm{Y} \mathrm{L}_{23}-$ edge as we will demonstrate in this example.

Figure 3 shows the EEL-spectrum of a thin $\mathrm{YBa}_{2} \mathrm{Cu}_{3} \mathrm{O}_{7}$-crystal $(t / \lambda=0.20)$. The relevant ionization edges used for the quantification are easily visible. Since the $\mathrm{Y} \mathrm{L}_{23}$-edge occurs at much higher energies than the other ionization edges, we recorded two spectra and spliced them together carefully. The backgrounds in front of the ionization edges were again fitted with the power-law model using a fitting region of $70 \mathrm{eV}$ for the $\mathrm{O} \mathrm{K}-, \mathrm{Ba} \mathrm{M}_{45}$ and the $\mathrm{Cu}_{23}$-edges and $150 \mathrm{eV}$ for the $\mathrm{Y} \mathrm{L}_{23}$-edge. The quantification results (Tab. II) show again that experimental $k$-factors yield the best agreement with the nominal composition. Particularly, for the $\mathrm{O} / \mathrm{Cu}$-ratio the hydrogenic model and the Hartree-Slater model result in values that are too high.

4.3 $\mathrm{LaB}_{6}$. - This material has been chosen to demonstrate that the experimental cross-sections obtained from oxides can be also applied to the quantification of other compounds such as borides, carbides and nitrides as well.

Figure 4 shows the original EEL-spectrum of $\mathrm{a} \mathrm{LaB}_{6}$-crystal $(t / \lambda=0.33$, not deconvoluted). Again backgrounds in front of the edges were fitted with the power-law model $\left(A E^{-r}\right)$ using a region of 30 to $40 \mathrm{eV}$ ahead of the edge onset. The quantification results are presented in Table II. If the $\mathrm{B} / \mathrm{La}$ ratio is determined using experimental $k$-factors, it is very close (5.45) to the nominal ratio of 6.0. The small deviation can be attributed to two main effects: first, it may result from a small oxygen signal (not shown in the spectrum), because oxygen may replace boron in 


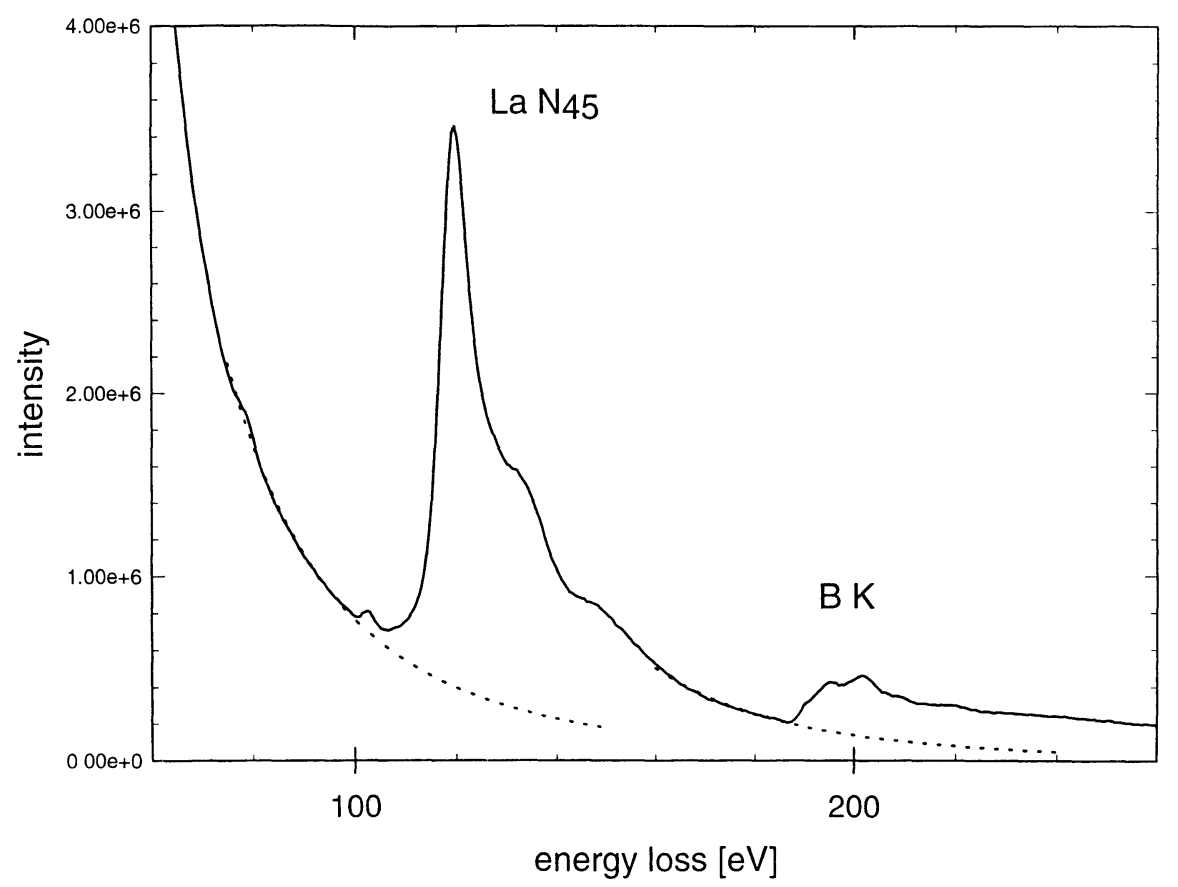

Fig. 4. - EEL spectrum of a thin $\mathrm{LaB}_{6}$-crystal; $(t / \lambda=0.35)$; background fits are indicated.

the boride easily. However, it is not clear at the moment if the oxygen found is an impurity or if it is caused by a thin oxidation layer on the surface of the specimen. Second, systematic errors introduced by different near-edge fine structures affect quantification as well, since $k$-factors have been determined from oxides and are applied to borides in this case. However, quantifications with calculated cross-sections result in a substantially larger deviation from the nominal ratio than experimental $k$-factors.

\section{Discussion}

In this work, we have shown that EELS should not be longer regarded as a qualitative tool. Accurate quantifications with a relative error of about $5 \%$ can be obtained if experimentally derived $k$-factors are applied.

Although the $k$-factors used for the quantifications have been measured under different experimental conditions $\left(E_{0}=120 \mathrm{kV}, \beta=5.9 \mathrm{mrad}\right)$ than the experimental spectra of this work $\left(E_{0}=200 \mathrm{kV}, \beta=7.6 \mathrm{mrad}\right)$, accurate quantifications are possible. This can be achieved by conversion of experimental $k$-factors to $200 \mathrm{kV}$ via the integrated dipole oscillator strengths with a short computer program [30].

A severe problem for EELS-quantification lies in the white lines of the $\mathrm{L}_{23}$-edges of the elements $\mathrm{K}$ to $\mathrm{Cu}$ and the $\mathrm{M}_{45}$-edges of the elements $\mathrm{Cs}$ to $\mathrm{Yb}$. The intensities of these white lines change according to the valency of the element specific edge and the white line to continuum ratio may be altered as well. Since the theoretical models cannot predict these white lines accurately, several workers proposed to subtract them from the experimental spectrum and to quantify only the continuum part. Since, however, the white lines are important analytically useable features, we cannot recommend this procedure. According to the findings of this work and previous results [35] 
Table III. - Recommended models for the calculation of ionization cross-sections giving fairly accurate EELS-quantifications; for usage if experimental $k$-factors are not available; based on previously published comparisons of models with experiments [13-15].

\begin{tabular}{||c||c||c||}
\hline \hline Edge type & Elements & Recommended model for ionization cross-sections \\
\hline \multirow{2}{*}{$\mathrm{K}$} & $\mathrm{Li}-\mathrm{Si}$ & H.S. model or Hydr. model (SIGMAK) [36] \\
\hline \multirow{2}{*}{$\mathrm{L}_{23}$} & $\mathrm{Al}-\mathrm{Ar}$ & H.S. model or Hydr. model (SIGMAL2) \\
\cline { 2 - 3 } & $\mathrm{K}-\mathrm{Zn}$ & Hydr. model (SIGMAL2) with white lines \\
\cline { 2 - 3 } & $\mathrm{Ga}-\mathrm{Br}$ & H.S. model \\
\cline { 2 - 3 } & $\mathrm{Rb}-\mathrm{Mo}$ & H.S. model \\
\hline \multirow{2}{*}{$\mathrm{M}_{45}$} & $\mathrm{Rb}-\mathrm{Cd}$ & Hydr. model [28] \\
\cline { 2 - 3 } & $\mathrm{In}-\mathrm{I}$ & H.S. model [28] \\
\cline { 2 - 3 } & $\mathrm{Cs}-\mathrm{Yb}$ & H.S. model (version without white lines) \\
\cline { 2 - 3 } & $\mathrm{Lu}-\mathrm{W}$ & H.S. model \\
\hline $\mathrm{N}_{45}$ & $\mathrm{Cs}-\mathrm{Yb}$ & experimental values only \\
\hline $\mathrm{M}_{23}$ & $\mathrm{~K}-\mathrm{Cu}$ & experimental values only \\
\hline
\end{tabular}

the white lines portion of the $\mathrm{L}_{23}$-edges in the hydrogenic model should not be removed: in case of the newer version of the hydrogenic model (SIGMAL2) fairly good quantifications for $\mathrm{L}_{23}$-edges can be obtained, because the white lines are taken into account, at least to some extent [13]. However, the older version without white line correction (SIGMAL1) gives less accurate quantification results. While the hydrogenic model enables good quantifications of the $\mathrm{K}$ - and $\mathrm{L}_{23}$-edges, there are severe problems for the higher ionization edges, e.g. $\mathrm{M}_{45}$ - or $\mathrm{N}_{45}$-edges. In case of the Hartree-Slater model the situation is slightly different: the Hartree-Slater model gives accurate quantifications for K-edges and many $\mathrm{L}_{23}$-edges, but problems may occur if ionization edges with white lines have to be quantified, because these features are not considered by this model. For example, the $\mathrm{L}_{23}$-edges of the elements $\mathrm{K}$ to $\mathrm{Cu}$ are better quantified by using the simple SIGMAL2 model (including the white lines!) instead of the Hartree-Slater model. Although the HS-model in its original form does not consider white lines, the overall match for the $\mathrm{M}_{45}$-edges is better than with the hydrogenic model.

In case of $\mathrm{N}_{45}$ - and $\mathrm{M}_{23}$-edges the calculated cross-sections deviate from the experimental crosssections by a factor of ten or even more, but these errors cancel out if two ionization edges of this type ( $\mathrm{N}_{45}$ - or $\mathrm{M}_{23}$-edges) are to be quantified. However, if such an edge has to be measured relative to e.g. a K-edge substantial errors will occur.

In all these situations experimental $k$-factors will give better accuracy because the $k$-factors are directly derived from experimental spectra, thus giving generally a better agreement with the edge profiles of the spectrum which has to be quantified. This is especially true for oxides, because $k$-factors have been obtained from oxide standards. Due to the close similarity of near-edge fine structures and overall edge shapes the agreement is then better among oxides, but a systematic error may be introduced in case of borides, carbides or nitrides. However, even then this error is lower compared to the deviations using calculated cross-sections. Although experimental values are available for almost every element and edge and should be favoured, it is sometimes necessary to have atomic theory cross-sections and profiles as well. 
In addition we include a table (Tab. III) giving recommendations about the models that may be used for quantitative EELS of different edge types. The models indicated for particular edge types and elements should yield results that lay within roughly $10-20 \mathrm{rel} \%$ of the nominal composition. These recommendations rely on previously published comparisons of calculated cross-sections with experiments [13-15].

\section{Acknowledgements}

We are very grateful to Prof. G. Herzog (TU Graz, Austria), Dr. A. Macher (TU Graz; Austria) and Dr. B. Freytag (University of Bonn; Germany) for providing test samples. We gratefully acknowledge financial support by the Forschungsförderungsfonds der Gewerblichen Wirtschaft, Vienna.

\section{References}

[1] Egerton R.F., Electron Energy-Loss Spectroscopy in the Electron Microscope, 2nd edition (Plenum Press, New York \& London, 1996).

[2] Leapman R.D. and Newbury D.E., Anal. Chem. 65 (1993) 2409.

[3] Ahn C.C. and Krivanek O.L., ASU/Gatan EELS Atlas, Center for Solid State Science (Arizona State University, Tempe, AZ 85281, U.S.A., 1983).

[4] Browning N.D., Chisholm M.F. and Pennycook S.J., Nature 366 (1993) 143.

[5] Tence M., Quartuccio M. and Colliex, C., Ultramicroscopy 58 (1995) 42.

[6] Liu D.R. and Williams D.B., Proc.45th Ann. EMSA Meeting, G.W. Bailey, Ed. (San Francisco Press Inc., San Francisco, 1987) p. 118.

[7] Wilson A.R., Microsc. Microanal. Microstruct. 2 (1991) 269.

[8] Leapman R.D., Rez P. and Mayers D.F., J. Chem. Phys. 72 (1980) 1232.

[9] Rez P., Ultramicroscopy 28 (1989) 16.

[10] Egerton R.F., Ultramicroscopy 4 (1979) 169.

[11] Egerton R.F., Proc. 39th Ann. EMSA Meeting, G.W. Bailey, Ed. (Claitor's Publishing, Baton Rouge, Lousiana, 1981) p. 198.

[12] Hofer F., Ultramicroscopy 21 (1987) 63.

[13] Hofer F., Microsc. Microanal. Microstruct. 2 (1991) 215.

[14] Egerton R.F., Ultramicroscopy 50 (1993) 13.

[15] Hofer F., in "Energy-Filtering Transmission Electron Microscopy", L. Reimer, Ed. (Springer-Verlag Berlin Heidelberg, New York, 1995) pp. 225-268.

[16] Hofer F. and Kothleitner G., Microsc. Microanal. Microstruct. 4 (1993) 539.

[17] Krivanek O.L., Gubbens A.J. and Dellby N., Microsc. Microanal. Microstruct. 2 (1991) 315.

[18] Krivanek O.L., Kundmann M.K., Trevor C. and Leapman R.D., in "Microbeam Analysis-1995, Proc. 29th Ann. Conf. Microbeam Analysis Society, Breckenridge, CO, E.S. Etz, Ed. (VCH Publishers Inc.) pp. 305-306.

[19] Egerton R.F., Williams B.G. and Sparrow T.G., Proc. Roy. Soc. Lond. A398 (1985) 3953.

[20] Egerton R.F. and Wang Z.L., Ultramicroscopy 32 (1990) 137.

[21] Bonnet N., Colliex C., Mory C. and Tence M., Scanning Microscopy Suppl. 2, Scanning Microscopy International, Chicago (AMF O'Hare, IL 60666, USA, 1988) p. 351.

[22] Shuman H. and Somlyo A.P., Ultramicroscopy 21 (1987) 23. 
[23] Leapman R.D. and Swyt C.R., Ultramicroscopy 26 (1988) 393.

[24] Manoubi T., Tence M., Walls M.G. and Colliex C., Microsc. Microanal. Microstruct. 1 (1990) 23.

[25] Press W.H., Flannery B.P., Teukolsky S.A. and Vetterling W.T., Numerical Recipes in Pascal-The Art of Scientific Computing (Cambridge University Press, 1989).

[26] Leapman R.D. and Hunt J.A., Microsc. Microanal. Microstruct. 2 (1991) 231.

[27] Brydson R., EMSA Bull. 21 (1991) 57.

[28] Luo B.P. and Zeitler E., J. Electr. Spectr. Rel. Phenom. 57 (1991) 285-295.

[29] Rez D. and Rez P., Microsc. Microanal. Microstruct. 3 (1992) 433-443.

[30] Egerton R.F., Proc. 46th Ann. Meeting EMSA, G.W. Bailiey, Ed. (San Francisco Press, San Francisco, 1988) pp. 532-533.

[31] Hofer F. and Wilhelm P., Ultramicroscopy 49 (1993) 189.

[32] Hofer F., Kothleitner G. and Rez P., Ultramicroscopy 63 (1996) 239.

[33] Macher A., Thesis, Technical University of Graz (1994).

[34] Karpinski J., Kaldis E., Jilek E. and Bucher B., Nature 336 (1988) 660.

[35] Hofer F. and Luo B., Ultramicroscopy 38 (1991) 159.

[36] Egerton R.F., Ultramicroscopy 63 (1996) 11. 